\title{
Research on Comprehensive Vulnerability of Grid Assessment Method Based on the Method of AHP-Grey Relation Degree
}

\author{
Shaoqian Ding ${ }^{1, a}$ \\ ${ }^{1}$ School of Electrical Engineering, Wuhan University, Wuhan, China \\ adingshaoqian1@163.com
}

\begin{abstract}
Keywords: power system; comprehensive vulnerability; analytic hierarchy process; grey relation degree; vulnerability assessment; vulnerable node.
\end{abstract}

\begin{abstract}
Based on the improved electrical betweenness and voltage stability theory, considering the importance of the structure of the grid and the current operational state of the power system, a comprehensive vulnerability assessment model and index based on the method of AHP-grey relation degree is introduced. Aiming at the shortcomings of only considering one of the structural vulnerability and state vulnerability assessment model, integrated expert's subjective opinion and objective data information, the comprehensive vulnerability assessment model can generally describe the vulnerable characteristics of assessment system, and scientifically identify weak nodes in the system. Finally based on the simulation of IEEE39 node system, it is verified the rationality and validity of the assessment index.
\end{abstract}

\section{Introduction}

For decades, many blackouts happened at home and abroad, which lead to experts and scholars from different countries for the different levels of analysis of the reasons of blackout[1-4]. The scale of power system blackouts usually expands rapidly because of certain components running out, which play an important role in the development of blackouts. Considering node failure will directly affect the normal operation of the branches, with which connected the node, node vulnerability is usually important than branch vulnerability.

Research tendency of the current power system vulnerability assessment methods from a single structure vulnerability assessment gradually to the comprehensive direction,which combined structural vulnerability and state vulnerability [5-10]. The literature [6] introduced betweenness and load economic factor to establish vulnerability assessment model combined structural vulnerability and state vulnerability, this integrated approach is simple and rough. The literature [9] introduced AHP method to research on comprehensive vulnerability, but it only consider subjective expert opinion, which is very likely to result in decision-maker's subjectivity.

In this paper, based on the improved electrical betweenness and voltage stability theory, considering the importance of the structure of the grid and the current operational state of the power system, a comprehensive vulnerability assessment model and index based on the method of AHP-grey relation degree is introduced. Analytic hierarchy process (AHP) uses the method of exponential scale, which both has order preserving, can guarantee the consistency of judgment matrix, and the accuracy is higher. Grey relation degree method give the corresponding weights based on structure vulnerability and state vulnerability index data. Integrated expert's subjective opinion and objective data information, the comprehensive vulnerability assessment model can generally describe the vulnerable characteristics of assessment system, and scientifically identify weak nodes in the system.

\section{The Index of Comprehensive Vulnerability}

In this paper, a comprehensive vulnerability index combined the AHP and grey relation degree is established. To overcome the shortcomings of the methods to subjectively or objectively determine the weight of indexes, improved AHP and grey correlation degree method is used to determine the 
weighting coefficient and to solve the problem of the selection of the weighting coefficient. The method of AHP is used to implement to calculate the weight coefficient between layer and layer in the system of vulnerability. The grey correlation method is used to modify the proportion made by experts based on the results of vulnerability index. As well as to a certain extent, it reduces the disturbance of the subjectivity, and can avoid completely based on the data, and avoid that there are great discrepancies in the assessment results and the recognition results.

From the paper [16], Based on the electrical betweenness index of the system circuit $B_{l_{k}}$, the concept of the node degree is introduced, the structural vulnerability index considering the active power and reactive power based on the improved electrical betweenness of node $i$ is proposed as equation (1):

$$
\omega_{s i}=\left|\sum_{k=1,2 \ldots n} B_{I_{k}}\right| .
$$

Wherein, $n$ represents the total number of lines connected to the node $i$. $B_{l_{k}}$ represents the structural vulnerability index of line $l_{k}$, which connected with the node $i$.

From the paper $[9,11]$, the state vulnerability index of node $i$ is proposed as equation (2):

$\omega_{r i}=\frac{1}{Y_{i}}=\frac{V_{c r}}{V_{i}-V_{c r}}$.

Wherein, $\quad Y_{i}$ is margin between the actual operating voltage and the critical voltage, $V_{i}$ s the operating voltage of node $i, V_{c r}$ is the critical voltage of load node.

Before the establishment of a comprehensive index, we need to normalize structural vulnerability indicators and state vulnerability indicators respectively, as shown in the equation (3).

$$
\left\{\begin{array}{l}
\tilde{\omega}_{s i}=\frac{\omega_{s i}-\omega_{s i_{-} \min }}{\omega_{s i_{-} \max }-\omega_{s i_{-} \min }} \\
\tilde{\omega}_{r i}=\frac{\omega_{r i}-\omega_{s i_{-} \min }}{\omega_{r i_{-} \max -\omega_{s i \_\min }}}
\end{array}\right.
$$

Wherein, $\tilde{\omega}_{s i} 、 \tilde{\omega}_{r i}$ represent the index of structural vulnerability and state vulnerability of node i respectively after the normalization. By the equation (3), $0 \leq \tilde{\omega}_{s i} \leq 1$, when the $\omega_{s i}=\omega_{s i \_m a x}, \tilde{\omega}_{s i}=1$, when the $\omega_{s i}=\omega_{s i \_\min }, \tilde{\omega}_{s i}=0$. Similarly, $0 \leq \tilde{\omega}_{r i} \leq 1$.

Concluded from the equation (3), the greater comprehensive vulnerability index is, the higher the importance of the node is. Conversely, it indicates that the relative safe of the node in the grid is.

In the analytic hierarchy process (AHP) [12-13], the scale is the method of quantitative calibration of the importance of the subgoal among each other. The most common of scale methods $1 \sim 9$ scale $9 / 9 \sim 9 / 1$ scale、 $2^{0 / 2} \sim 2^{8 / 2}$ scale 、and $e^{0 / 4} \sim e^{8 / 4}$ scale methods, and so on. In this paper, considering factors such as the consistency, the rank preservation scale uniformity, fitting scale weight of the construction judgment matrix, So choose $e^{0 / 4} \sim e^{8 / 4}$ index scale method to construct judgment matrix, as shown in table 1.

Tab.1 Exponent scale of judgment matrix

\begin{tabular}{lccccc}
\hline assessment & $\begin{array}{c}\text { Equally } \\
\text { important }\end{array}$ & $\begin{array}{c}\text { Small } \\
\text { important }\end{array}$ & $\begin{array}{c}\text { Slightly } \\
\text { important }\end{array}$ & $\begin{array}{c}\text { More } \\
\text { important }\end{array}$ & $\begin{array}{c}\text { Obviously } \\
\text { important }\end{array}$ \\
\hline Scale alues & $e^{0 / 4}$ & $e^{1 / 4}$ & $e^{2 / 4}$ & $e^{3 / 4}$ & $e^{4 / 4}$ \\
\hline assessment & $\begin{array}{c}\text { Very } \\
\text { important }\end{array}$ & $\begin{array}{c}\text { Strongly } \\
\text { important }\end{array}$ & $\begin{array}{c}\text { More } \\
\text { Strongly } \\
\text { important }\end{array}$ & $\begin{array}{c}\text { Extremely } \\
\text { important }\end{array}$ & \\
\hline Scale alues & $e^{5 / 4}$ & $e^{6 / 4}$ & $e^{7 / 4}$ & $e^{8 / 4}$ & \\
\hline
\end{tabular}


This paper adopts AHP method to construct the comprehensive objective function by making the structural vulnerability index and state vulnerability index as target data fusion, then the weight of structural vulnerability index and state vulnerability index $\xi_{1} 、 \xi_{2}$ can be obtained, which depends on the the important degree in the vulnerability assessment system.

In the process of using the method of grey relation degree to determine the corresponding weight, structural vulnerability and state vulnerability are identified as assessment indexes at the same time. in order to amend corresponding weighting domains of comprehensive evaluation by the change of objective conditions.

Dynamic weight of characteristic parameter's formulas calculated by the method of grey relation degree method are as follows[14-15]:

1) Determine the assessment indexes and the initial weight value. Assuming that m experts make a experience judgment for the weights of $n$ assessment indexes at the same time, the matrix $A$ can be obtained.

2) Determine the reference sequence $A_{0}$.

$A_{0}=\left(a_{01}, a_{02}, \cdots a_{0 m}\right)$.

3) Calculate the distance of each index series $A_{1}, A_{2}, \cdots, A_{n}$ between the reference sequence $A_{0}$, see the formula (5).

$D_{0 i}=\sum_{k=1}^{m}\left(a_{0 k}-a_{1 k}\right)^{2}$.

4) Calculate the weights of the indexes, see the formula (6).

$\zeta_{i}=1 /\left(1+D_{0 i}\right)$.

Through the calculation of pure numerical method, on the basis of subjective information making full use of expert experience judgment value, the grey relation degree method guarantees the objectivity of the calculation process, the weight can be got, which reflect the degree of subjective and objective.

the comprehensive vulnerability indexes which is determined based AHP- Grey relation degree method are shown as equation (7).

$R_{j}=\xi_{j} * \zeta_{j}$.

The structure vulnerability and state vulnerability after normalization are substituted into the comprehensive vulnerability index by weighted form, as shown in the formula (8). Finally normalize the comprehensive vulnerability indexes by the formula (3).

$\tau=\mathrm{R}_{1} * \tilde{\omega}_{s i}+\mathrm{R}_{2} * \tilde{\omega}_{r i} \cdot$

Wherein, $R_{1} 、 R_{2}$ are the weighting of the structural vulnerability index and state vulnerability index respectively. The weights of comprehensive vulnerability index can be combined with specific examples to assign the judging matrix, to establish comprehensive vulnerability index base on the specific examples and operation manner.

Grid vulnerability is not only related to running state of the system, but also it was influenced by the network topology features,comprehensive vulnerability index take these influences into consideration, it can characterize and evaluate vulnerability more comprehensively.

\section{Numerical Examples}

Choose the IEEE -39 node system as example of analysis, which the specific parameters in literature [17]. 


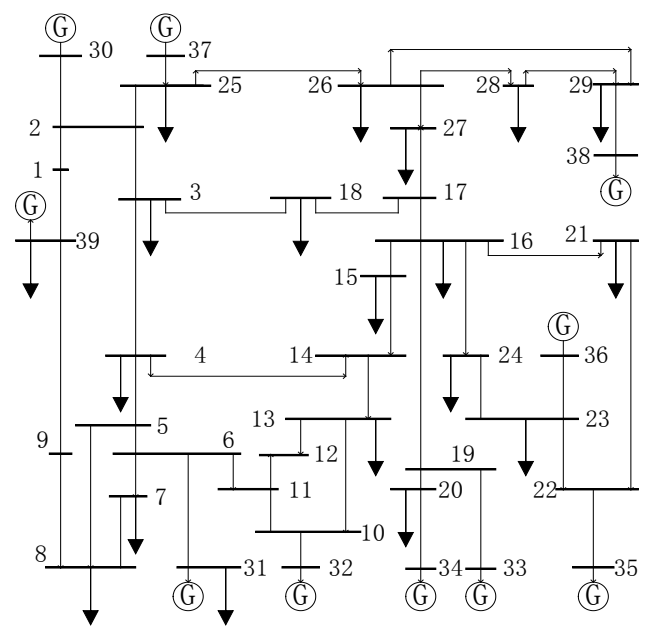

Fig.1 Diagram of IEEE-39 node power system

Tests on the standard IEEE 39 bus system, and sorts the results in accordance with a comprehensive vulnerability in descending order. The sorted results are shown in Table 2.

Tab.2 The sort of load nodes' comprehensive vulnerability

\begin{tabular}{cccc}
\hline ranking & $\begin{array}{c}\text { structural } \\
\text { vulnerability }\end{array}$ & $\begin{array}{c}\text { state } \\
\text { vulnerability }\end{array}$ & $\begin{array}{c}\text { Comprehensive } \\
\text { vulnerability }\end{array}$ \\
\hline 1 & 16 & 12 & 16 \\
2 & 8 & 15 & 15 \\
3 & 4 & 28 & 8 \\
4 & 15 & 4 & 4 \\
5 & 3 & 8 & 7 \\
6 & 7 & 7 & 12 \\
7 & 18 & 16 & 27 \\
8 & 20 & 27 & 18 \\
9 & 27 & 18 & 3 \\
10 & 21 & 21 & 28 \\
11 & 23 & 23 & 21 \\
12 & 29 & 25 & 20 \\
13 & 25 & 3 & 23 \\
14 & 24 & 20 & 25 \\
15 & 12 & 29 & 29 \\
16 & 26 & 24 & 24 \\
17 & 28 & 26 & 26 \\
\hline
\end{tabular}

From the above table, it can be seen that the top 3 ranking of comprehensive vulnerability index of load nodes are node 16, node 15 and node 3 . The node 16 is taken as an example to illustrate. It is important in the grid structure, and closely linked to other nodes in the network, so it has the highest index of structure vulnerability. The state vulnerability index of node 16 is 7 th. Through the comprehensive vulnerability assessment based on the improved AHP-Grey relation degree method it ranks for the first. The comprehensive vulnerability index of node 12 is 6th of all load nodes. It is slightly terminal in IEEE-39 node power system, and its structural vulnerability index among all load nodes ranks 15th. But because the running state of node 12 is very vulnerable, and its running state is approaching critical state, the ranking of state vulnerability ranks for the first, so the ranking of comprehensive vulnerability rose to sixth, which verify the vulnerability of nodes in the system is decided by the importance in the grid structure and the operational state.

The minimum of comprehensive vulnerability index is node 26. It carries lighter load. If the node occurs failure and even quit running will not hurt the integrity of the whole network structure, so its structural vulnerability index is very small. And node 26 is close to generator node 37, which the generator node provides power support to ensure the running state of node 26 stable and reliable. So its comprehensive vulnerability is minimal, the method described in this article can comprehensively evaluate the comprehensive vulnerability of load nodes. 


\section{Summary}

It proposes in this paper a comprehensive vulnerability assessment model based on improved AHP- Grey relation degree method. Considering the importance of the structure of the grid and the current operational state of the power system, the comprehensive vulnerability assessment model applies the theory of AHP-Grey relation degree method in the study of vulnerability. Integrating subjective opinions of experts and objective data information about the node, the method can effectively identify and assess potential weaknesses, scientifically evaluate the ability to resist high risk cascading failure in power grid. Finally it verifies the rationality and effectiveness of the assessment method by IEEE39 node system. The simulation results show that the assessment method can generally describe the vulnerable characteristics of assessment system, and scientifically identify weak nodes in the system.

\section{About the Author:}

Ding Shaoqian received the B.S.E.E. degree in Electric Engineering from HeBei University of Science \& technology, China in 2012. Now she is studying in the school of Electrical Engineering at Wuhan University for M.E. degree, Wuhan, China. Her research interests include power system operation and control. Email:dingshaoqian1@163.com.

\section{References}

[1] National Power Dispatching and Communication Center 《guide of the power system security and stability 》 learning and counseling [M]. Beijing: China Electric Power Press, 2001,81-83.

[2] C W Taylor. Improving grid behavior.IEEE Spectrum.1999,36(6):40-45.

[3] Carson W.Taylor, Dennis C. Erickson. Recording and Analyzing the July 2 Cascading Outage IEEE Computer Applications in Power,1999,10(1):26-30.

[4] He Dayu .Rethinking over '8.14’US-CANADA Blackout after one year [J].Power System Technology , 2004,28 (21): 1-5.

[5] Ding Jian, Bai Xiaomin, Zhao Wei and so on. Grid Vulnerability Analysis Based on Two-dimensional Accumulation Means [J].Automation of Electric Power Systems, 2008,32 (8): 1-4.

[6] Weizhen Bo, LiuJunyong. A New Integrative Vulnerability Evaluation Model to Power Grid Based on Running State and Structure [J].Automation of Electric Power Systems,2009, 33 (8): 11-14.

[7] Nixiang Ping, Mei Shengwei, Zhang Xuemin. Transmission Lines Vulnerability Assessment Based on Complex Network Theory[J]. Automation of Electric Power Systems, 2008,32 (4): 1-5.

[8] Zhang Guohua, Zhang Jianhua, Yang Jingyan, and so on.Vulnerability assessment of bulk power grid based on weighted directional graph and complex network theory [J]. Electric Power Automation Equipment, 2009,29 (4): 21-26.

[9] Xie Chunwei, Fang Fei, and so on. Research on Power Grid Vulnerability Assessment Methods [J]. Power System and Clean Energy, 2013,29 (5): 35-38.

[10] Liang Yueming, Xie Zicheng. Node Vulnerability Assessment on power grid Based Information Entropy and Hierarchy process[J].Journal of Electric Power,2013,28 (3): 196-200.

[11] Deng Guiping, Sun Yuanzhang, Xu Jian. A new voltage stability analysis method by considering short circuit capacity[J]. Automation of Electric Power Systems, 2009, 33(8): 15-19.

[12] Changjiane, JiangTaili. research Determine the weights by Analytic Hierarchy Process[J]. Wuhan University of Technology, 2007,29 (1) : 153-15. 
[13] WANG Rui, FANG Jie, ZHANG Ke, et al. Fuzzy synthetic evaluation of power quality based on entropy and AHP[J] . Electrical Measurement \& Instrumentation, 2007, 44(11) : 21-25.

[14] ZHANG Xinjie, GE Shaoyun, LIU Hong. Comprehensive Assessment System and Method of Smart Distribution Grid [J]. Power System Technology, 2014, 38(1) : 40-46.

[15] XU Hang, LI Huaqiang, ZHAO Xiangyun, et al.Assessment on Nodal Comprehensive Vulnerability Based on Operational State and Network Structure[J] . Power System Technology, 2014, 38(3) : 731-735.

[16] Liu Yaonian ,Zhu Xi,YuBing, Gong Wei-guo, Zhang Wei-min Analysis of Power System Vulnerability Based on Electrial Betwenness [J].Electrical Measurement \& Instrumentation. 2011,48(547):61-63.

[17] Wang Fang, " Studies on Methods of Network Partitioning for Voltage Reactive Power Control Based on Short-circuit Impedance Distance and Reactive Power Margin," MasterDegree. dissertation, Dept. Electrical Engineering. Eng., Univ. Chongqing,2013. 